\section{P266 TOWARDS CLOUD COMPUTING AS A PLATFORM FOR SUPPORTING HIV INFORMATION DISSEMINATION IN UGANDA: A CASE OF JSCS AND TASO KAMPALA}

Cliffford Benoni*. British Computer Society, Kampala, Uganda

10.1136/sextrans-2019-sti.394

Background The use of ICT to improve the dissemination of HIV awareness information should be a feature of everyday life, in a disease prone Uganda today, being a developing country with high prevalence of HIV/AIDs. It is, therefore, paramount that ICT policies that support the dissemination of HIV/AIDs information are put in place to adopt new concepts and technologies that create new avenues. Cloud computing is viewed as a potential technology infrastructure that can be used to improve efficiency and effectiveness of operations geared towards health information dissemination. However, it is yet to be embraced. Our aim was to: Find out the major platforms of HIV/AIDS information dissemination today in Uganda. Investigate the management of cloud computing technology in HIV/AIDs information dissemination in lieu of the existing technologies. Identify challenges, affordability and accessibility of cloud computing in Uganda.

Methods Literature review and interaction with health information providers both in public and private hospitals were done. Data was collected using questionnaires, interviews and observation.

Results The study found out that the adoptability of the technology is bedevilled by lack of local service providers, lack of technical personnel and fear of hosting sensitive data, outside the borders of Uganda. The technology was, however, found to be very relevant both in government and private sector health care services in supporting the increase and effective HIV/AIDS information dissemination. The study created awareness on the existence of a government cloud and recommended a new brokerage model that can be used for the creation and dissemination of information.

Conclusion The potential and impact of cloud computing is undoubtedly quantifiable, especially, for Ugandan hospitals and practitioners that run on low budgets. The model identified in the study can be used in individual attendee environments.

Disclosure No significant relationships.

\section{P270 HEALTH RIGHTS: LGBTIQ COMMUNITY}

Tyrone Havnar*. I Reach Out My Hand Africa, Human Resources, Harare, Zimbabwe

10.1136/sextrans-2019-sti.395

Background The Zimbabwean HIV epidemic is largely driven by unprotected heterosexual sex. Now there is a growing epidemic among key populations who are at higher risk of HIV. National data is sparse. Only a minimal amount of data is collected and reported in national documents. The KP is disproportionately burdened by HIV infection, situation which is worsened by laws that penalise same-sex intercourse and contribute to a cycle of stigma, homonegativity and discrimination. African countries' laws criminalising homosexuality may be fuelling the epidemic; they dissuade KPs from seeking treatment and health care providers from offering it.
Zimbabwe is one of the countries where homosexuality is ontra, onos, ores. The hostile environment the KP community is exposed to especially at health facilities in the country has impacted negatively on their rights to basic SRHR. Some have been keeping sexually transmitted infections for months without seeking help. Such discrimination and stigma at the highest level makes life difficult and remain secretive and isolated community always fearing for lives. Zimbabwe's Constitution promotes universal access to health enabling every person regardless of their sexual orientation to be treated with respect and have access to healthcare and support. The everyday reality though is very different. We have held sensitisation workshops with stakeholders to root out ignorance and misinformation associated with the LGBTI community. Hostility and beliefs systems deep rooted against the practise of same sex relationship in the country will need to be reversed. While Zimbabwe's Constitution stipulates healthcare for all, it also outlaws same sex marriages. The gay community continues to be marginalised making the fight against HIV/AIDS all the more difficult. The intersectionality of HIV/AIDS between the broader heterosexuals and LGBTI community is a reality. If we are to reduce or end new infections end deaths from AIDS end stigma and discrimination in Zimbabwe no one should be left behind.

Methods To increase testing particularly among hard to reach groups is self-testing. In 2015 Population Services International and UNITAID began HIV Self Testing Africa (STAR), a four-year project to scale up self-testing in Zimbabwe Malawi and Zambia. In the first year nearly 380000 free HIV self-test kits were distributed in 27 districts in Malawi, Zambia and Zimbabwe. Results suggest self-testing is enabling more young people (aged 16-24 years) and men to be aware of their HIV status. In the first year young people comprised $28 \%$ of self-tests and resulted in testing coverage among this age group increasing by $39 \%$ in the project's catchment areas. Men accounted for $44 \%$ of self-test users and testing coverage increased by $28 \%$ in testing areas. Among those using the kits in Zimbabwe $23 \%$ were first-time testers. Homosexual acts are illegal in Zimbabwe for men who have sex with men (MSM) but legal for women who have sex with women.

Results With the help of mobilizers the identified 300 MSM \& $280(62 \%)$ participated in a survey. Over $100(38 \%)$ reported they never reported testing for HIV leaving 120 for analysis. 200 men used the HIVST. HIV prevalence was $6 \%$ (14/200). Overall (55\%) were between 21 and 30 years-old, received less than a college-level education (54\%) an annual income of $\$ 3400(48 \%)$. Majority of men identified as gay (84\%) never married (93\%), only a third disclosed sexual contact with other men to healthcare providers (28\%), (75) had tested for HIV in the past 3 months. The common venue for seeking sex partners was the internet (90\%).104 men (32\%) had sex under the influence of alcohol/drugs within the last 3 months, 42 men (12\%) engaged in group sex in the past year, 42 received payment for sex with money or gifts.

Conclusion As a consequence of this punitive law national statistics are rarely available. Criminalising men who have sex with men drives this vulnerable group away from HIV services. As a result, many do not know their HIV status let alone access treatment. However Zimbabwean organisations 